\title{
New Gedanken experiment on RN-AdS black holes surrounded by quintessence
}

\author{
Yang $Q u^{\mathrm{a}}$, Jun Tao ${ }^{\mathrm{b}}$, Jiayi $\mathrm{Wu}^{\mathrm{c}}$ \\ Center for Theoretical Physics, College of Physics, Sichuan University, Chengdu 610065, China
}

Received: 10 August 2021 / Accepted: 10 February 2022 / Published online: 1 March 2022

(C) The Author(s) 2022

\begin{abstract}
In this paper, we use the new version of Gedanken experiment to investigate the weak cosmic censorship conjecture(WCCC) for RN-AdS black holes surrounded by quintessence. The process of matter fields falling into the black hole can be regarded as a dynamic process. Since the perturbation of matter fields doesn't affect the spacetime geometry, we propose the stability condition and assume the process of matter fields falling into the black hole satisfies the null energy condition. Based on the stability condition and the null energy condition, the first-order and secondorder perturbation inequalities are derived. As a result, we show that the WCCC for RN-AdS black holes surrounded by quintessence cannot be violated under the second-order approximation of matter fields perturbation.
\end{abstract}

\section{Introduction}

The most fundamental prediction of general relativity is the existence of black holes. Black holes have event horizon and a gravitational singularity inside the event horizon. If the event horizon vanishes, the naked singularity will destroy the well-define spacetime and the law of causality. To solve this problem, Penrose [1] proposed the weak cosmic censorship conjecture (WCCC), which indicates the singularity should be hidden in the event horizon and the observer at infinity cannot receive any information from the singularity.

Even though there is no general method to prove this conjecture, many efforts have been made to test it. The Gedanken experiment was firstly proposed by Wald [2] In 1974 to verify the applicability of WCCC in extremal Kerr-Newman (KN) black holes. The experiment shows that the conjecture will not be destroyed under the first-order perturbation when considering a particle with enough charges (or angular

\footnotetext{
a e-mail: quyang@stu.scu.edu.cn

b e-mail: taojun@scu.edu.cn (corresponding author)

ce-mail: wujiayi777@stu.scu.edu.cn
}

momentum) falling towards the extremal $\mathrm{KN}$ black holes. Over the last few years, many similar works based on this experiment have been considered in different works [3-13]. But there are some inherent flaws in this approach. It only considers the process of particles falling into black holes but does not consider the background spacetime's effect on the test particle and the analysis only focus on the first-level perturbation of the process. Later, Hubery [14] considered the second-order perturbation of test particles to show that the $\mathrm{KN}$ black holes can be destroyed in the Gedanken experiment. After that, the WCCC for other kinds of black holes is examined, and it's found that the conjecture can be violated during the process [15-23].

To deal with these defects, Sorce and Wald [24] proposed the new version of the Gedanken experiment, which considers matter fields instead of particles. Then an assumption indicates that if the matter fields fall into the black holes after a long time, the configuration of spacetime should be the same as the original one. Based on Iyer-Wald formalism [25] and null energy condition, the second-order perturbation inequality is derived to show that WCCC is valid under the second-order approximation of the matter fields perturbation. After that, many works based on this new experiment [26-41] show that WCCC is applicable for other kinds of black holes. In addition, more study on the test for WCCC can be found in [42-60].

Observations over the last century have shown that the universe is dominated by an energetic component with the negative pressure $[61,62]$. One of the candidates for this component is the quintessence, which is a slow-changing and spatially non-uniform component of the negative pressure [6368]. The quintessence is described by an ordinary scalar field minimally coupled to gravity, whose potential leads to the expansion. Quintessence must be coupled to ordinary matter, which results in long-range interaction and time dependent natural constants, even if it's compressed to Planck's scale. Kiselev [69] proposed the typical dark energy with black 
holes to explain this phenomenon. Since then, relevant studies have been conducted successively [70-72]. We consider the contribution of the space-time gravitational field of extra energy, the momentum tensor, which consists of dark energy. Then we can study the thermodynamic effects of black holes surrounded by the dark energy [73-84]. Next, we will consider the thermodynamics of the black hole surrounded by quintessence and use the Gedanken experiment to test the WCCC.

The organization of the paper is as follows. In Sect. 2, we study the spacetime geometry of RN-AdS black holes surrounded by quintessence under the matter fields perturbation. In Sect. 3, we discuss the Iyer-Wald formalism and derive the first-order and second-order variational identities. In Sect. 4, based on the variational identities and null energy condition, we derive the first-order and the second-order perturbation inequalities. In Sect. 5 we use the first-order optimal option and the second-order perturbation inequality to examine the WCCC for nearly extremal RN-AdS black holes surrounded by quintessence. In Sect. 6, some discussions and conclusions are given.

\section{Spacetime gemogery of $\mathrm{RN}$-AdS black hole surrounded by quintessence}

In this paper, we consider the RN-AdS black holes surrounded by quintessence in four-dimensional spacetime. The Lagrangian of this case could be described as follows [69,83]

$L=\frac{1}{16 \pi}\left(R-2 \Lambda-F_{a b} F^{a b}+\mathcal{L}_{q}\right) \varepsilon$,

where $F=d A$ is the strength of the electromagnetic field and $A$ is the potential of the electromagnetic field. $\Lambda$ is the cosmological constant with a negative value, $\varepsilon$ is the volume element, $\mathcal{L}_{q}$ is the quintessence dark energy such as a barotropic perfect fluid and defined by [85]

$\mathcal{L}_{q}=-\rho_{q}\left[1+\omega \ln \frac{\rho}{\rho_{0}}\right]$,

where $\rho_{0}$ is an integral constant, $\rho_{q}$ is the energy density, and $\omega$ is the quintessence dark energy barotropic index. The value range of $\omega$ is $-1<\omega<-\frac{1}{3}$ for the quintessence dark energy.

We use the Eddingdon-Finkelstein coordinate in this work [35]. Compared with Schwarzschild gauge, there is no coordinate singularity at the event horizon. When we consider the matter fields following into the black hole, it is convenient to use this coordinate to calculate. The solution of RN-AdS black holes surrounded by quintessence is

$$
d s^{2}=-f(r) d v^{2}+2 d r d v+r^{2}\left(d \theta^{2}+\sin ^{2} \theta d \phi^{2}\right),
$$

where

$f(r)=1-\frac{2 M}{r}+\frac{Q^{2}}{r^{2}}-\frac{\Lambda r^{2}}{3}-\frac{a}{r^{3 \omega+1}}$.

here $M$ and $Q$ can be interpreted as the mass and charge of the black hole respectively, $l$ is the radius of $\mathrm{AdS}$ space and is related to the cosmological constant by $\Lambda=-\frac{3}{l^{2}}$. The parameter $a$ is the normalization factor associated with the quintessence density of dark energy and it's always positive. The relationship between $a$ and $\rho_{q}$ can be expressed as [75, 77]

$\rho_{q}=-\frac{a}{2} \frac{3 \omega}{r^{3(\omega+1)}}$.

By imposing the condition $f(r)=0$, we can derive the radius of event horizon $r_{+}$. Based on the first law of thermodynamics in the extended space $[75,86]$, we can obtain that

$P=-\frac{\Lambda}{8 \pi}, \quad V_{+}=\frac{4}{3} \pi r_{+}^{3}, \quad \mathcal{A}_{+}=-\frac{1}{2 r_{+}^{3 \omega}}$,

where $P$ is the thermodynamic pressure, $V$ is the thermodynamic volume, and $\mathcal{A}$ is conjugate to the parameter $a$.

The process of matter fields falling into the black hole can be considered as a completely dynamic process. We further assume that cosmological constant and normalization factor $a$ can be regarded as effective parameters determined by the matter source coupled to the Einstein-Maxwell gravity. Then, we can represent the Lagrangian as

$L=\frac{1}{16 \pi}\left(R-F_{a b} F^{a b}\right) \varepsilon+L_{m t}$.

$L_{m t}$ is Lagrangian of matter field, and the spacetime geometry can be simply derived from the Lagrangian if the matter field satisfies the stable solution. Then we can obtain the energy-momentum tensor of matter fields [83],

$T_{a b}=\frac{\Lambda}{8 \pi} g_{a b}-\rho_{q} g_{a b}$.

Based on the Iyer-Wald formalism [24], $n$-form Lagrangian can be regarded as the function of metric $g_{a b}$ and other tensor fields $\psi$ in spacetime. We use $\varphi$ to represent the collection of the dynamical field, i.e. $\boldsymbol{\varphi}=\left(g_{a b}, \psi\right)$. In this case, we set $\varphi$ to be the collection of dynamical field $\varphi=\left(g_{a b}, A\right)$ and use $\lambda$ to express the perturbation of the matter field to $\phi$. The spacetime field equations are

$$
\begin{aligned}
& R_{a b}(\lambda)-\frac{1}{2} R(\lambda) g_{a b}(\lambda)=8 \pi\left(T_{a b}^{E M}(\lambda)+T_{a b}(\lambda)\right), \\
& \nabla_{a}^{(\lambda)} F^{b a}(\lambda)=4 \pi j^{b}(\lambda),
\end{aligned}
$$

where $T_{a b}^{E M}$ is the energy-momentum tensor of electromagnetic field. 
Considering the perturbation process of matter fields, the spacetime geometry can be described as [35]

$$
\begin{aligned}
d s^{2}= & -f(r, v, \lambda) d v^{2}+2 \mu(r, v, \lambda) d r d v \\
& +r^{2}\left(d \theta^{2}+\sin ^{2} \theta d \phi^{2}\right) .
\end{aligned}
$$

We assume that the spacetime satisfies the stability condition [24], which means that after enough long time, the spacetime geometry should be consistent with the RN-AdS black hole surrounded by quintessence. We utilize $M, Q, \Lambda$ to describe the properties of black holes and represent these quantities by the parameter $\lambda$. Thus, the dynamical fields can be expressed as [35]

$$
\begin{aligned}
& d s^{2}=-f(r, \lambda) d \nu^{2}+2 d r d \nu+r^{2}\left(d \theta^{2}+\sin ^{2} \theta d \phi^{2}\right) \\
& F(\lambda)=\boldsymbol{d} \boldsymbol{A}, A(\lambda)=-\frac{Q(\lambda)}{r} d \nu
\end{aligned}
$$

where

$f(r, \lambda)=1-\frac{2 M(\lambda)}{r}+\frac{Q^{2}(\lambda)}{r^{2}}-\frac{\Lambda(\lambda) r^{2}}{3}-\frac{a(\lambda)}{r^{3 \omega+1}}$.

The energy-momentum tensor of matter fields is

$T_{a b}(\lambda)=\left[\frac{\Lambda(\lambda)}{8 \pi}-\rho_{q}(\lambda)\right] g_{a b}(\lambda)$.

When the parameter $\lambda$ is zero, the spacetime should still be the solution of RN-AdS black holes surrounded by quintessence, i.e. when $f(r, 0)=f(r)$, metric can be restored to the original spacetime form.

\section{The linear variational identities}

In this paper, we would like to use the Iyer-Wald formalism $[24,25]$ to investigate the Gedanken experiments in RNAdS black holes surrounded by quintessence. Consider the Einstein-Maxwell Theory, the Lagrangian is expressed as

$L=\frac{1}{16 \pi}\left(R-F_{a b} F^{a b}\right) \varepsilon$.

We use $\boldsymbol{\varphi}=\left(g_{a b}, A\right)$ to represent the collection of the dynamical field. The variation of Lagrangian is

$\delta L=E_{\varphi} \delta \varphi+d \Theta(\varphi, \delta \varphi)$,

where $E_{\varphi}=0$ gives the equations of motion. $\Theta$ is the threeform of the symplectic potential and is locally composed of $\varphi$ and its derivatives. In the Einstein-Maxwell theory, $\Theta$ can be linearly expressed with gravitational field part and electromagnetic field part as

$$
\begin{aligned}
& \Theta_{a b c}^{G R}(\phi, \delta \phi)=\frac{1}{16 \pi} \varepsilon_{d a b c} g^{d e} g^{f g}\left(\nabla_{g} \delta g_{e f}-\nabla_{e} \delta g_{f g}\right), \\
& \Theta_{a b c}^{E M}(\phi, \delta \phi)=-\frac{1}{4 \pi} \varepsilon_{d a b c} F^{d e} \delta A_{e} .
\end{aligned}
$$

Then we define the three-form of symplectic current as

$\omega\left(\boldsymbol{\varphi}, \delta_{1} \boldsymbol{\varphi}, \delta_{2} \varphi\right)=\delta_{1} \Theta\left(\boldsymbol{\varphi}, \delta_{2} \varphi\right)-\delta_{2} \Theta\left(\varphi, \delta_{1} \varphi\right)$.

It also can be linearly expressed by two parts,

$$
\begin{aligned}
\omega_{a b c}^{G R} & =\frac{1}{16 \pi} \varepsilon_{d a b c} \eta^{d}, \\
\omega_{a b c}^{E M} & =\frac{1}{4 \pi}\left(\delta_{2}\left(\varepsilon_{d a b c} F^{d e}\right) \delta_{1} A_{e}-\delta_{1}\left(\varepsilon_{d a b c} F^{d e}\right) \delta_{2} A_{e}\right),
\end{aligned}
$$

where

$\eta^{a}=p^{a b c d e f}\left(\delta_{2} g_{b c} \nabla_{d} \delta_{1} g_{e f}-\delta_{1} g_{b c} \nabla_{d} \delta_{2} g_{e f}\right)$,

with

$$
\begin{aligned}
p^{a b c d e f}= & g^{a e} g^{f b} g^{c d}-\frac{1}{2} g^{a d} g^{b e} g^{f c}-\frac{1}{2} g^{a b} g^{c d} g^{e f} \\
& -\frac{1}{2} g^{b c} g^{a e} g^{f d}+\frac{1}{2} g^{b c} g^{a d} g^{e f} .
\end{aligned}
$$

For an arbitrary vector field, the Noether current associated with $\zeta^{a}$ is defined by

$J_{\zeta}=\Theta\left(\varphi, \mathscr{L}_{\zeta} \varphi\right)-\zeta \cdot L$

The variation of Noether current [25] is

$$
\begin{aligned}
\delta J_{\zeta}= & -\zeta \cdot(E(\varphi) \cdot \delta \varphi)+\omega\left(\boldsymbol{\varphi}, \delta \varphi, \mathscr{L}_{\zeta} \varphi\right) \\
& +d(\Theta(\zeta \cdot \boldsymbol{\varphi}, \delta \varphi)) .
\end{aligned}
$$

As shown in Ref. [87], we can write the Noether current as

$J_{\zeta}=C_{\zeta}+d Q_{\zeta}$

where $Q_{\zeta}$ is the Noether charge and $C_{\zeta}=\zeta^{a} C_{a}$ is the constraint to the theory. We can obtain $C_{a}=0$ and $d J_{\zeta}=0$ from the equation of motion. The Noether charge $Q_{\zeta}$ is linearly expressed by

$Q_{\zeta}=Q_{\zeta}^{G R}+Q_{\zeta}^{E M}$,

where

$$
\begin{aligned}
& \left(Q_{\zeta}^{G R}\right)_{a b}=-\frac{1}{16 \pi} \varepsilon_{a b c d} \nabla^{c} \zeta^{d}, \\
& \left(Q_{\zeta}^{E M}\right)_{a b}=-\frac{1}{8 \pi} \varepsilon_{a b c d} F^{c d} A_{e} \zeta^{e} .
\end{aligned}
$$

Considering the Einstein-Maxwell Theory, the equations of motion and constraints are given by

$$
\begin{aligned}
E_{\varphi} \delta \varphi & =-\varepsilon\left(\frac{1}{2} T^{a b} \delta g_{a b}+j^{a} \delta A_{a}\right), \\
C_{a b c d} & =\varepsilon_{e b c d}\left(T_{a}^{e}+A_{a} j^{e}\right),
\end{aligned}
$$

where $T_{a b}=\frac{1}{8 \pi} G_{a b}-T_{a b}^{E M}$ and $j^{b}=\frac{1}{4 \pi} \nabla_{a} F^{b a}$ are the energy-momentum tensor and electric current respectively.

By differentiating Eq. (23) and substituting Eq. (22), we can obtain the first-order variational identity,

$$
\begin{aligned}
& d\left(\delta Q_{\zeta}-\zeta \cdot \Theta(\boldsymbol{\varphi}, \delta \varphi)\right) \\
& \quad=\omega\left(\boldsymbol{\varphi}, \delta \boldsymbol{\varphi}, \mathscr{L}_{\zeta} \boldsymbol{\varphi}\right)-\zeta \cdot E_{\varphi} \delta \varphi-\delta C_{\zeta} .
\end{aligned}
$$


In the same way, by differentiating Eq.(27), the second-order variational identity can be shown as

$$
\begin{aligned}
& d\left(\delta^{2} Q_{\zeta}-\zeta \cdot \delta \Theta(\boldsymbol{\varphi}, \delta \boldsymbol{\varphi})\right) \\
& \quad=\omega\left(\boldsymbol{\varphi}, \delta \boldsymbol{\varphi}, \mathscr{L}_{\zeta} \delta \boldsymbol{\varphi}\right)-\zeta \cdot \delta E_{\varphi} \delta \varphi-\delta^{2} C_{\zeta} .
\end{aligned}
$$

\section{First-order and second-order perturbation inequalities}

In this section, we calculate the integral of first-order and second-order variational identities to obtain the perturbation inequalities. Due to the stability condition, we can choose a hypersurface $\Sigma=H \cup \Sigma_{1}$. $H$ is a portion of the horizon $r=r_{+}$in the background spacetime, starting from the unperturbed horizon's bifurcate surface $B$ and ending up on the cross-section $B_{1} . \Sigma_{1}$ approaches infinity along the timeslice ( $v=$ constant) as a space-like hypersurface. And the event horizon is a Killing horizon generated by the Killing field $\zeta^{a}$. For the first-order variational equation, we utilize the condition $\mathscr{L}_{\zeta} \varphi=0$ and integrate it on the hypersurface $\Sigma$

$$
\begin{aligned}
& \int_{\Sigma} d\left(\delta Q_{\zeta}-\zeta \cdot \Theta(\varphi, \delta \varphi)\right) \\
& \quad+\int_{\Sigma} \zeta \cdot E_{\varphi} \delta \varphi+\int_{\Sigma} \delta C_{\zeta}=0 .
\end{aligned}
$$

Using the Stokes theorem and the condition that $B$ is unperturbed horizon's bifurcate surface, it can be rewritten as

$$
\begin{aligned}
& \int_{S_{o}}\left(\delta Q_{\zeta}-\zeta \cdot \Theta(\varphi, \delta \varphi)\right) \\
& \quad+\int_{\Sigma_{1}} \zeta \cdot E_{\varphi} \delta \varphi+\int_{\Sigma_{1}} \delta C_{\zeta}+\int_{H} \delta C_{\zeta}=0 .
\end{aligned}
$$

Since the integral diverges as the integral region approaches infinity, we apply a cut-off method at sphere $S_{o}$ with radius $r_{o}$ and let the limit of $S_{o}$ approach asymptotic infinity.

Then, we calculate each integration term separately. Firstly, we evaluate the first term of Eq. (30). Considering Eqs. (11), (16) and (25), we have

$$
\begin{aligned}
\int_{S_{o}} & (\delta Q-\zeta \cdot \Theta(\boldsymbol{\varphi}, \delta \boldsymbol{\varphi})) \\
= & \int_{S_{o}}\left(\delta Q^{G R}-\zeta \cdot \Theta^{G R}(\boldsymbol{\varphi}, \delta \boldsymbol{\varphi})\right) \\
& +\int_{S_{o}}\left(\delta Q^{E M}-\zeta \cdot \Theta^{E M}(\boldsymbol{\varphi}, \delta \boldsymbol{\varphi})\right) \\
= & \delta M-V_{o} \delta P-\mathcal{A}_{o} \delta a-\frac{1}{8 \pi} \int_{S_{o}} \epsilon_{a b c d} A_{e} \zeta^{e} \delta F^{c d}
\end{aligned}
$$

As the $S_{e}$ approaching asymptotic infinity, the second term vanishes, and then the first term of Eq. (30) is given by

$\int_{S_{o}}\left(\delta Q_{\zeta}-\zeta \cdot \Theta(\boldsymbol{\varphi}, \delta \varphi)\right)=\delta M-V_{o} \delta P-\mathcal{A}_{o} \delta a$.

Secondly, we integrate the second part of Eq. (30) by considering $\varphi(0)$ as a globally hyperbolic, asymptotically flat solution of the equations of motion, which means that the integral of the second term equal to zero [24].

Then, we utilize the condition $j^{a}=0$ on $\Sigma_{1}$ and Eq. (26) to show that

$\int_{\Sigma_{1}} \delta C_{\zeta}=\left(V_{o}-V_{+}\right) \delta P+\left(\mathcal{A}_{o}-\mathcal{A}_{+}\right) \delta a$,

where

$V_{e}=\frac{4}{3} \pi r_{o}^{3}, \quad \mathcal{A}_{o}=-\frac{1}{2 r_{o}^{3 \omega}}$.

Finally, with $\left.A_{a} \zeta^{a}\right|_{H}=-\Phi_{+}$and $\int_{H} \varepsilon_{e b c d} \delta j=\delta Q$, we can further obtain the expression as

$\int_{H} \delta C_{\zeta}=\int_{H} \varepsilon_{e b c d} \zeta^{a} \delta T_{a}^{e}-\Phi_{+} \delta Q$.

Since both the normal vectors $n^{a}$ and the time-like Killing vectors $\zeta^{a}$ on the horizon become null and $\zeta^{a} \propto n^{a}$, we can use the null energy condition $\delta T_{a b} n^{a} n^{b} \geqslant 0$. On the horizon, we have $\varepsilon_{e b c d}=-4 n_{[e} \widetilde{\varepsilon}_{b c d]}$, where $n^{a}$ is the normal vector and $\widetilde{\varepsilon}_{b c d}$ is the volume element, so the first term of Eq. (35) can be written as $\int_{H} \widetilde{\varepsilon}_{b c d} n_{e} \zeta^{a} \delta T_{a}^{e}(\lambda) \geqslant 0$. Therefore, from Eqs. $(32,33,35)$ and the null energy condition, we can obtain the first-order perturbation inequality

$\delta M-\Phi_{+} \delta Q-V_{+} \delta P-\mathcal{A}_{+} \delta a \geqslant 0$.

In our work, we use the second-order perturbation inequality to examine whether the WCCC can be violated under the second-order perturbation approximation of matter fields. When the first-order inequality is satisfied, it can be proved that WCCC is valid under the first-order approximation. But when the first-order perturbation takes the optimal option

$\delta M-\Phi_{+} \delta Q-V_{+} \delta P-\mathcal{A}_{+} \delta a=0$,

the WCCC can not be examined sufficiently by only considering the first-order approximation. Then we need to derive the second-order perturbation inequality.

Integrating Eq. (28) on the hypersurface $\Sigma$, it yields

$$
\begin{aligned}
& \int_{S_{o}} \delta\left(\delta Q_{\zeta}-\zeta \cdot \Theta(\boldsymbol{\varphi}, \delta \boldsymbol{\varphi})\right) \\
& \quad+\int_{\Sigma} \delta\left(\zeta \cdot E_{\varphi} \delta \varphi\right)+\int_{\Sigma_{1}} \delta^{2} C_{\zeta}+\int_{H} \delta^{2} C_{\zeta} \\
& \quad-\mathscr{W}_{H}(\boldsymbol{\varphi}, \delta \boldsymbol{\varphi})-\mathscr{W}_{\Sigma_{1}}(\boldsymbol{\varphi}, \delta \boldsymbol{\varphi})=0,
\end{aligned}
$$


where

$$
\begin{aligned}
\mathscr{W}_{H}(\boldsymbol{\varphi}, \delta \varphi) & =\int_{H} \omega\left(\boldsymbol{\varphi}, \delta \boldsymbol{\varphi}, \mathscr{L}_{\zeta} \delta \boldsymbol{\varphi}\right), \\
\mathscr{W}_{\Sigma_{1}}(\boldsymbol{\varphi}, \delta \varphi) & =\int_{\Sigma_{1}} \omega\left(\boldsymbol{\varphi}, \delta \boldsymbol{\varphi}, \mathscr{L}_{\zeta} \delta \boldsymbol{\varphi}\right) .
\end{aligned}
$$

Following the previous calculation steps, the second term of Eq. (38) equals to 0, the third and fourth term of Eq. (38) can be expressed respectively

$$
\begin{aligned}
\int_{\Sigma_{1}} \delta^{2} C_{\zeta} & =\left(V_{e}-V_{+}\right) \delta^{2} P+\left(\mathcal{A}_{e}-\mathcal{A}_{+}\right) \delta^{2} a, \\
\int_{H} \delta^{2} C_{\zeta} & =\int_{H} \varepsilon_{e b c d} \zeta^{a} \delta^{2} T_{a}^{e}(\lambda)-\Phi_{+} \delta^{2} Q .
\end{aligned}
$$

Similarly, the first term can be written as

$$
\begin{aligned}
\int_{S_{o}} \delta(\delta Q-\zeta \cdot \Theta(\boldsymbol{\varphi}, \delta \boldsymbol{\varphi})) \\
=\int_{S_{o}} \delta\left(\delta Q^{G R}-\zeta \cdot \Theta^{G R}(\boldsymbol{\varphi}, \delta \boldsymbol{\varphi})\right) \\
\quad+\int_{S_{o}} \delta\left(\delta Q^{E M}-\zeta \cdot \Theta^{E M}(\boldsymbol{\varphi}, \delta \boldsymbol{\varphi})\right) \\
=\int_{S_{o}} \delta\left(\delta Q^{G R}-\zeta \cdot \Theta^{G R}(\boldsymbol{\varphi}, \delta \boldsymbol{\varphi})\right) \\
\quad-\frac{1}{8 \pi} \int_{S_{o}} \epsilon_{a b c d} \delta A_{e} \zeta^{e} \delta F^{c d} \\
-\frac{1}{8 \pi} \int_{S_{o}} \epsilon_{a b c d} A_{e} \zeta^{e} \delta^{2} F^{c d} \\
=\delta^{2} M-V_{o} \delta^{2} P-A_{o} \delta^{2} a \\
\quad-\frac{1}{8 \pi} \int_{S_{o}} \epsilon_{a b c d} \delta A_{e} \zeta^{e} \delta F^{c d}
\end{aligned}
$$

where the second term on the last line vanishes if $S_{e}$ approaches asymptotic infinity, and then the first term of Eq. (38) is given by

$$
\int_{S_{o}} \delta Q_{\zeta}-\zeta \cdot \Theta(\varphi, \delta \varphi)=\delta^{2} M-V_{o} \delta^{2} P-\mathcal{A}_{o} \delta^{2} a .
$$

Finally, we calculate the fifth term of Eq. (38). It can be linearly expressed by two parts

$\mathscr{W}_{H}=\int_{H} \omega^{G R}+\int_{H} \omega^{E M}$.

From [24], we can obtain the similar result, and then we have

$\mathscr{W}_{H}=\int_{H} \varepsilon_{e b c d} \zeta^{a} \delta^{2} T_{a}^{e E M}$.

Therefore, from Eqs. (40), (41), and (44), we can get

$$
\begin{aligned}
& \delta^{2} M-\Phi_{+} \delta^{2} Q-V_{+} \delta^{2} P-\mathcal{A}_{+} \delta^{2} a \\
& \quad=\mathscr{W}_{\Sigma_{1}}(\boldsymbol{\varphi}, \delta \boldsymbol{\varphi})-\int_{H} \varepsilon_{e b c d} \zeta^{a} \delta^{2}\left(T_{a}^{e E M}+T_{a}^{e}\right) .
\end{aligned}
$$

Following the same method in Ref. [24], we can build an auxiliary spacetime to calculate. Because of the stability condition, the spacetime geometry on $\Sigma_{1}$ is still the RN-AdS spacetime surrounded by quintessence, and the configuration of dynamical fields under the perturbation of matter field can be described by one parameter $\lambda$. Hence, the metric function and electromagnetic strength for the auxiliary spacetime are

$$
\begin{gathered}
d s_{Q R}^{2}(\rho)=-f^{Q R}(r, \lambda) d v^{2}+2 d r d v \\
+r^{2}\left(d \theta^{2}+\sin ^{2} \theta d \phi^{2}\right), \\
F=\frac{Q^{Q R}(\lambda)}{r^{2}} d r \wedge d v,
\end{gathered}
$$

where

$$
\begin{aligned}
f^{Q R}(r, \lambda)= & 1-\frac{2 M^{Q R}(\lambda)}{r}-\frac{\Lambda^{Q R}(\lambda) r^{2}}{3} \\
& +\frac{\left(Q^{Q R}(\lambda)\right)^{2}}{r^{2}}-\frac{a^{Q R}(\lambda)}{r^{3 \omega+1}} .
\end{aligned}
$$

Next we only consider the first-order variation of matter field on auxiliary spacetime, and then the $M^{R A}(\lambda), Q^{R A}(\lambda)$, $\Lambda^{R A}(\lambda)$ and $a^{Q R}(\lambda)$ are given as follows,

$$
\begin{gathered}
M^{Q R}(\lambda)=M+\lambda \delta M, \quad Q^{Q R}(\lambda)=Q+\lambda \delta Q, \\
\Lambda^{Q R}(\lambda)=\Lambda+\lambda \delta \Lambda, \quad a^{Q R}(\lambda)=a+\lambda \delta a .
\end{gathered}
$$

Considering only the first-order variation of the matter field, we obtain $\delta^{2} M^{Q R}=\delta^{2} Q^{Q R}=\delta^{2} \Lambda^{Q R}=\delta^{2} a^{Q R}=0$. Then we obtain $\delta \varphi^{Q R}=\delta \varphi$ on hypersurface $\Sigma_{1}$, which implies that $\mathscr{W}_{\Sigma_{1}}(\varphi, \delta \varphi)=\mathscr{W}_{\Sigma_{1}}\left(\varphi, \delta \varphi^{Q R}\right)$. Thus we can calculate them straightly in auxiliary spacetime. have

Integrating the second-order variation identity on $\Sigma_{1}$, we

$$
\mathscr{W}_{\Sigma_{1}}\left(\boldsymbol{\varphi}, \delta \varphi^{Q R}\right)=\int_{\partial \Sigma_{1}} \delta\left(\delta Q_{\zeta}^{Q R}-\zeta \cdot \Theta\left(\varphi^{Q R}, \delta \varphi^{Q R}\right)\right) .
$$

Following the previous calculation steps and the gauge condition of the electromagnetic field such that $\zeta^{a} \delta A_{a}=0$ at $H$, the Eq. (49) can be expressed by

$\mathscr{W}_{\Sigma_{1}}\left(\varphi, \delta \varphi^{Q R}\right)=\frac{1}{4 \pi} \int_{B_{1}} \frac{\delta Q^{2}}{r} \sin \theta d \theta d \phi=\frac{\delta Q^{2}}{r_{+}}$.

Then, the Eq. (45) can be rewritten as

$$
\begin{gathered}
\delta^{2} M-\Phi_{+} \delta^{2} Q-V_{+} \delta^{2} P-\frac{\delta Q^{2}}{r_{+}}-\mathcal{A}_{+} \delta^{2} a \\
=-\int_{H} \varepsilon_{e b c d} \zeta^{a} \delta^{2}\left(T_{a}^{e E M}+T_{a}^{e}\right) .
\end{gathered}
$$

We consider the null energy condition $\delta^{2}\left(T_{a b}^{E M}+T_{a b}\right) n^{a} n^{b}$ $\geqslant 0$. under the second-order approximation. Then the second-order perturbation inequality can be reduced as

$\delta^{2} M-\Phi_{+} \delta^{2} Q-V_{+} \delta^{2} P-\frac{\delta Q^{2}}{r_{+}}-\mathcal{A}_{+} \delta^{2} a \geqslant 0$. 


\section{Test the WCCC of RN-AdS black hole surrounded by quintessence}

In this section, we will apply the new version of the Gedanken experiment to discuss the WCCC of nearly extremal RNAdS black holes surrounded by quintessence. We assume that the spacetime satisfies the stability condition. The condition of the existing event horizon $r_{+}$is metric factor satisfy $f\left(r_{+}\right)=0$. We suppose that there exists one minimum point at $r=r_{0}$ for $f(r)$, and the existence of the event horizon is consistent with condition $f\left(r_{0}\right) \leqslant 0$. We can take $f\left(r_{0}(\lambda), \lambda\right)$ and use the discriminant function to represent the change of extremum of $f(r)$ under the matter field perturbation. $r_{0}(\lambda)$ is the minimum point of $f\left(r_{0}(\lambda), \lambda\right)$, which satisfies the condition $\partial_{r} f\left(r_{0}(\lambda), \lambda\right)=0$. We can expand the function to second-order at $\lambda=0$,

$f\left(r_{0}(\lambda), \lambda\right) \simeq f\left(r_{0}, 0\right)+f^{\prime} \lambda+f^{\prime \prime} \frac{\lambda^{2}}{2}+\mathcal{O}\left(\lambda^{2}\right)$.

With $\partial_{r} f\left(r_{0}(\lambda), \lambda\right)=0$ and the zero-order approximation of $\lambda$, one can obtain

$M=\frac{6 Q^{2} r_{0}^{3 \omega-1}+2 \Lambda r_{0}^{3 \omega+3}-3(3 \omega+1) a}{6 r_{0}^{3 \omega}}$.

Considering the matter fields and taking the first-order variation of $\partial_{r} f\left(r_{0}(\lambda), \lambda\right)=0$, we have

$$
\begin{aligned}
\delta r_{0}= & \frac{2 r_{0}^{3 \omega+1}}{2 \Lambda r_{0}^{3 \omega+3}+3 \omega(3 \omega+1) a-2 Q^{2} r_{0}^{3 \omega-1}} \\
& \times\left[\delta M-\frac{2 Q \delta Q}{r_{0}}-\frac{\delta \Lambda r_{0}^{3}}{3}+\frac{(3 \omega+1) \delta a}{2 r_{0}^{3 \omega}}\right] .
\end{aligned}
$$

Therefore, by applying Eqs. (54) and (55), we can yield the detailed expression of Eq. (53) as

$$
\begin{aligned}
& f\left(r_{0}(\lambda), \lambda\right) \\
&=\frac{r_{0}^{3 \omega+1}-Q^{2} r_{0}^{3 \omega-1}-\Lambda r_{0}^{3 \omega+3}+3 \omega a}{r_{0}^{3 \omega+1}} \\
& \quad-\frac{2 \lambda}{r_{0}}\left(\delta M-\frac{Q \delta Q}{r_{0}}+\frac{\delta \Lambda r_{0}^{3}}{6}+\frac{\delta a}{2 r_{0}^{3 \omega}}\right) \\
& \quad-\frac{\lambda^{2}}{r_{0}}\left\{\delta^{2} M-\frac{Q \delta^{2} Q}{r_{0}}+\frac{\delta^{2} \Lambda r_{0}^{3}}{6}+\frac{\delta^{2} a}{2 r_{0}^{3 \omega}}\right. \\
&\left.+\left[r_{0} \Lambda+\frac{3 \omega(3 \omega+1) a}{2 r_{0}^{3 \omega+2}}-\frac{Q^{2}}{r_{0}^{3}}\right] \delta r_{0}^{2}\right\} \\
&+\lambda^{2}\left[\frac{\delta Q^{2}+2 \delta M \delta r_{0}}{r_{0}^{2}}-\frac{4 Q \delta Q \delta r_{0}}{r_{0}^{3}}-\frac{2 r_{0} \delta \Lambda \delta r_{0}}{3}\right. \\
&+\left.\frac{(3 \omega+1) \delta a \delta r_{0}}{r_{0}^{3 \omega+2}}\right] .
\end{aligned}
$$

The event horizon $r_{+}$and $r_{0}$ satisfy the relation $r_{+}(1-$ $\varepsilon)=r_{0}$ with $\varepsilon \ll 1$ [24] for the case of the nearly extremal black hole. With the relation of $\partial_{r} f\left(r_{0}\right)=0$, $f^{\prime}\left(r_{+}\right)=\varepsilon r_{+} f^{\prime \prime}\left(r_{+}\right)$, and we can obtain the relation of $f\left(r_{0}\right)=-\frac{1}{2} \varepsilon^{2} r_{+}^{2} f^{\prime \prime}\left(r_{+}\right)$under the second-order approximation of $\varepsilon$. We can conclude these relations,

$$
\begin{gathered}
\frac{r_{0}^{3 \omega+1}-Q^{2} r_{0}^{3 \omega-1}-\Lambda r_{0}^{3 \omega+3}+3 \omega a}{r_{0}^{3 \omega+1}} \\
\quad=\left[1-\frac{2 Q^{2}}{r_{+}^{2}}+\frac{3 \omega(3 \omega+3) a}{2 r_{+}^{3 \omega+1}}\right] \varepsilon^{2} .
\end{gathered}
$$

Therefore, we can rewrite the expression of Eq. (56) as

$$
\begin{aligned}
f\left(r_{0}(\lambda), \lambda\right) & \\
= & {\left[1-\frac{2 Q^{2}}{r_{+}^{2}}+\frac{3 \omega(3 \omega+3) a}{2 r_{+}^{3 \omega+1}}\right] \varepsilon^{2} } \\
& -\frac{\lambda \varepsilon}{r_{+}^{2}}\left(\delta M-\Phi_{+} \delta Q-V_{+} \delta P-\mathcal{A}_{+} \delta a\right) \\
& +\frac{\lambda \varepsilon}{r_{+}^{2}}\left(2 Q \delta Q+r_{+}^{4} \delta \Lambda-\frac{3 \omega \delta a}{r_{+}^{3 \omega-1}}\right) \\
& -\frac{\lambda^{2}}{r_{0}}\left\{\left(\delta^{2} M-\Phi_{+} \delta^{2} Q-V_{+} \delta^{2} P-\frac{\delta Q^{2}}{r_{+}}-\mathcal{A}_{+} \delta^{2} a\right)\right. \\
& \left.+\left[r_{0} \Lambda+\frac{3 \omega(3 \omega+1) a}{2 r_{0}^{3 \omega+2}}-\frac{Q^{2}}{r_{0}^{3}}\right] \delta r_{0}^{2}\right\} \\
& +\lambda^{2}\left[\frac{\delta Q^{2}+2 \delta M \delta r_{0}}{r_{0}^{2}}-\frac{4 Q \delta Q \delta r_{0}}{r_{0}^{3}}\right. \\
& \left.-\frac{2 r_{0} \delta \Lambda \delta r_{0}}{3}+\frac{(3 \omega+1) \delta a \delta r_{0}}{r_{0}^{3 \omega+2}}\right]
\end{aligned}
$$

Utilizing the Eqs. (37) and (52) together with above results, we can further simplify the expression as

$$
\begin{aligned}
& f\left(r_{0}(\lambda), \lambda\right) \\
& \leq\left[1-\frac{2 Q^{2}}{r_{+}^{2}}+\frac{3 \omega(3 \omega+3) a}{2 r_{+}^{3 \omega+1}}\right] \varepsilon^{2} \\
& \quad+\frac{\lambda \varepsilon}{r_{+}^{2}}\left(2 Q \delta Q+r_{+}^{4} \delta \Lambda-\frac{3 \omega \delta a}{r_{+}^{3 \omega-1}}\right) \\
& \quad+\frac{\lambda^{2}\left(-2 Q \delta Q r_{+}^{3 \omega-1}-\delta \Lambda r_{+}^{3 \omega+3}+3 \omega \delta a\right)^{2}}{2 r_{+}^{3 \omega+1}\left(2 \Lambda r_{+}^{3 \omega+3}+3 \omega(3 \omega+1) a-2 Q^{2} r_{+}^{3 \omega-1}\right)} .
\end{aligned}
$$

With the condition that $f\left((1+\varepsilon) r_{0}\right)=0$ and $f^{\prime}\left(r_{0}\right)=0$, and considering the zero-order approximation of $\varepsilon$, we can derive $\Lambda$ and $\mathrm{M}$, 


$$
\begin{aligned}
\Lambda & =\frac{r_{0}^{3 \omega+1}-Q^{2} r_{0}^{3 \omega-1}+3 \omega a}{r_{0}^{3 \omega+3}}, \\
M & =\frac{2 r_{0}^{3 \omega+1}+4 Q^{2} r_{0}^{3 \omega-1}-3(\omega+1) a}{6 r_{0}^{3 \omega}} .
\end{aligned}
$$

Together with the relation $r_{+}=(1+\varepsilon) r_{0}$, we can expressed Eq. (59) as

$$
f\left(r_{0}(\lambda), \lambda\right) \leq-\frac{r_{0}^{3}(\varepsilon A+\lambda B)^{2}}{2 f^{\prime \prime}\left(r_{0}\right)}
$$

where

$$
\begin{aligned}
& A=9 a \omega^{2}+9 a \omega-4 Q^{2} r_{0}^{3 \omega-1}+2 r_{0}^{3 \omega+1}, \\
& B=2 Q \delta Q r_{0}^{3 \omega-1}+\delta \Lambda r_{0}^{3 \omega+3}-3 \omega \delta a .
\end{aligned}
$$

Because $r_{0}$ is the minimum point that satisfies the condition $f^{\prime \prime}\left(r_{0}\right)>0$. The above expression gives $f\left(r_{0}(\lambda), \lambda\right) \leqslant$ 0 , which implies that the event horizon of near extremal RNAdS black holes surrounded by quintessence still exists when the second-order perturbation is taken into account, therefore the WCCC cannot be violated under the second-order approximation of matter fields perturbation.

\section{Conclusion}

In this paper, we discuss the WCCC of nearly extremal RNAdS black holes surrounded by quintessence with the new version of Gedanken experiment. Based on the stability condition and the null energy condition, we utilize Iyer-Wald formalism to derive the first-order and the second-order perturbation inequalities. With the first-order optimal option and the second-order inequality, we prove that the event horizon of nearly extremal RN-AdS black holes surrounded by quintessence still exists under the second-order approximation of matter fields perturbation, which implies that the WCCC can not be violated for this case. This result is the same as that of previous papers [79-81]. Furthermore, we can prove the event horizon still exists under the higher perturbation or another black hole, this gives us a broader perspective and methods to examine the WCCC.

Acknowledgements We are grateful to Peng Wang, Wei Hong, and Siyuan Hui for useful discussions. This work is supported in part by NSFC (Grant Nos. 11947408 and 11875196 and 12047573).

Data Availability Statement This manuscript has no associated data or the data will not be deposited. [Authors' comment: This is a theoretical study and no experimental data.]

Open Access This article is licensed under a Creative Commons Attribution 4.0 International License, which permits use, sharing, adaptation, distribution and reproduction in any medium or format, as long as you give appropriate credit to the original author(s) and the source, provide a link to the Creative Commons licence, and indicate if changes were made. The images or other third party material in this article are included in the article's Creative Commons licence, unless indicated otherwise in a credit line to the material. If material is not included in the article's Creative Commons licence and your intended use is not permitted by statutory regulation or exceeds the permitted use, you will need to obtain permission directly from the copyright holder. To view a copy of this licence, visit http://creativecomm ons.org/licenses/by/4.0/.

Funded by $\mathrm{SCOAP}^{3}$.

\section{References}

1. R. Penrose, Gravitational collapse: the role of general relativity. Riv. Nuovo Cim. 1, 252-276 (1969)

2. R. Wald, Gedanken experiments to destroy a black hole. Ann. Phys. 82(2), 548-556 (1974)

3. J.M. Cohen, R. Gautreau, Naked singularities, event horizons, and charged particles. Phys. Rev. D 19, 2273-2279 (1979)

4. T. Needham, Cosmic censorship and test particles. Phys. Rev. D 22, 791-796 (1980)

5. I. Semiz, Dyon black holes do not violate cosmic censorship. Class. Quantum Gravity 7, 353-359 (1990)

6. I. Semiz, Dyonic Kerr-Newman black holes, complex scalar field and cosmic censorship. Gen. Relativ. Gravit. 43, 833-846 (2011)

7. J.D. Bekenstein, C. Rosenzweig, Stability of the black hole horizon and the Landau ghost. Phys. Rev. D 50, 7239-7243 (1994)

8. B. Gwak, Stability of horizon in warped AdS black hole via particle absorption. Results Phys. 13, 102155 (2019)

9. X.X. Zeng, X.Y. Hu, K.J. He, Weak cosmic censorship conjecture with pressure and volume in the Gauss-Bonnet AdS black hole. Nucl. Phys. B 949, 114823 (2019)

10. P. Wang, H. Wu, H. Yang, Thermodynamics of nonlinear electrodynamics black holes and the validity of weak cosmic censorship at charged particle absorption. Eur. Phys. J. C 79(7), 572 (2019)

11. B. Mu, J. Tao, P. Wang, Minimal length effect on thermodynamics and weak cosmic censorship conjecture in anti-de Sitter black holes via charged particle absorption. Adv. High Energy Phys. 2612946 (2020)

12. S. Isoyama, N. Sago, T. Tanaka, Cosmic censorship in overcharging a Reissner-Nordstrom black hole via charged particle absorption. Phys. Rev. D 84, 124024 (2011)

13. D. Chen, Thermodynamics and weak cosmic censorship conjecture in extended phase spaces of anti-de Sitter black holes with particles' absorption. Eur. Phys. J. C 79(4), 353 (2019)

14. V.E. Hubeny, Overcharging a black hole and cosmic censorship. Phys. Rev. D 59, 064013 (1999)

15. F. de Felice, Y.Q. Yu, Turning a black hole into a naked singularity. Class. Quantum Gravity 18, 1235-1244 (2001)

16. T. Jacobson, T.P. Sotiriou, Over-spinning a black hole with a test body. Phys. Rev. Lett. 103, 141101 (2009). [Erratum: Phys. Rev. Lett. 103, 209903 (2009)]

17. J.V. Rocha, R. Santarelli, Flowing along the edge: spinning up black holes in AdS spacetimes with test particles. Phys. Rev. D 89(6), 064065 (2014)

18. M. Bouhmadi-Lopez, V. Cardoso, A. Nerozzi, J.V. Rocha, Black holes die hard: can one spin-up a black hole past extremality? Phys. Rev. D 81, 084051 (2010)

19. S. Gao, Y. Zhang, Destroying extremal Kerr-Newman black holes with test particles. Phys. Rev. D 87(4), 044028 (2013)

20. K. Düztaş, Overspinning BTZ black holes with test particles and fields. Phys. Rev. D 94(12), 124031 (2016)

21. G. Chirco, S. Liberati, T.P. Sotiriou, Gedanken experiments on nearly extremal black holes and the Third Law. Phys. Rev. D 82, 104015 (2010) 
22. S. Hod, Cosmic censorship, area theorem, and selfenergy of particles. Phys. Rev. D 66, 024016 (2002)

23. A. Saa, R. Santarelli, Destroying a near-extremal Kerr-Newman black hole. Phys. Rev. D 84, 027501 (2011)

24. J. Sorce, R.M. Wald, Gedanken experiments to destroy a black hole. II. Kerr-Newman black holes cannot be overcharged or overspun. Phys. Rev. D 96(10), 104014 (2017)

25. V. Iyer, R.M. Wald, Some properties of Noether charge and a proposal for dynamical black hole entropy. Phys. Rev. D 50, 846-864 (1994)

26. J. An, J. Shan, H. Zhang, S. Zhao, Five-dimensional Myers-Perry black holes cannot be overspun in gedanken experiments, Phys. Rev. D 97(10), 104007 (2018)

27. Y.L. He, J. Jiang, Weak cosmic censorship conjecture in EinsteinBorn-Infeld black holes. Phys. Rev. D 100(12), 124060 (2019)

28. B. Ge, Y. Mo, S. Zhao, J. Zheng, Higher-dimensional charged black holes cannot be over-charged by gedanken experiments. Phys. Lett. B 783, 440-445 (2018)

29. B. Chen, F.L. Lin, B. Ning, Gedanken Experiments to Destroy a BTZ Black Hole. Phys. Rev. D 100(4), 044043 (2019)

30. J. Jiang, B. Deng, Z. Chen, Static charged dilaton black hole cannot be overcharged by gedanken experiments. Phys. Rev. D 100(6), 066024 (2019)

31. J. Jiang, X. Liu, M. Zhang, Examining the weak cosmic censorship conjecture by gedanken experiments for Kerr-Sen black holes. Phys. Rev. D 100(8), 084059 (2019)

32. J. Jiang, Static charged Gauss-Bonnet black holes cannot be overcharged by the new version of gedanken experiments. Phys. Lett. B 804, 135365 (2020)

33. X.Y. Wang, J. Jiang, Gedanken experiments at high-order approximation: nearly extremal Reissner-Nordstrom black holes cannot be overcharged. JHEP 05, 161 (2020)

34. J. Jiang, Y. Gao, Investigating the gedanken experiment to destroy the event horizon of a regular black hole. Phys. Rev. D 101(8), 084005 (2020)

35. X.Y. Wang, J. Jiang, Examining the weak cosmic censorship conjecture of RN-AdS black holes via the new version of the gedanken experiment. JCAP 07, 052 (2020)

36. B Chen, FL Lin, B Ning, Y Chen, Constraints on low-energy effective theories from weak cosmic censorship. Phys. Rev. Lett. 126(3), $031102(2021)$

37. J. Jiang, M. Zhang, Testing the weak cosmic censorship conjecture in Lanczos-Lovelock gravity. Phys. Rev. D 102(8), 084033 (2020)

38. J. Jiang, M. Zhang, New version of the gedanken experiments to test the weak cosmic censorship in charged dilaton-Lifshitz black holes. Eur. Phys. J. C 80(9), 822 (2020)

39. M. Zhang, J. Jiang, New gedanken experiment on higherdimensional asymptotically AdS Reissner-Nordström black hole. Eur. Phys. J. C 80(9), 890 (2020)

40. H.F. Ding, X.H. Zhai, Examining the weak cosmic censorship conjecture by gedanken experiments for an Einstein-MaxwellDilaton-Axion black hole. Mod. Phys. Lett. A 35(40), 2050335 (2020)

41. F. Qu, S.J. Yang, Z. Wang, J.R. Ren, Weak cosmic censorship conjecture is not violated for a rotating linear dilaton black hole. arXiv:2008.09950 [gr-qc]

42. T. Bai, W. Hong, B. Mu, J. Tao, Weak cosmic censorship conjecture in the nonlinear electrodynamics black hole under the charged scalar field. Commun. Theor. Phys. 72(1), 015401 (2020)

43. B. Gwak, Weak cosmic censorship conjecture in Kerr-(Anti-)de Sitter black hole with scalar field. JHEP 09, 081 (2018)

44. B. Gwak, Weak cosmic censorship with pressure and volume in charged anti-de sitter black hole under charged scalar field. JCAP 08, 016 (2019)

45. B. Gwak, Weak cosmic censorship in Kerr-Sen black hole under charged scalar field. JCAP 03, 058 (2020)
46. D. Chen, Weak cosmic censorship conjecture in BTZ black holes with scalar fields. Chin. Phys. C 44(1), 015101 (2020)

47. D. Chen, W. Yang, X. Zeng, Thermodynamics and weak cosmic censorship conjecture in Reissner-Nordstrom anti-de Sitter black holes with scalar field. Nucl. Phys. B 946, 114722 (2019)

48. S.J. Yang, J. Chen, J.J. Wan, S.W. Wei, Y.X. Liu, Weak cosmic censorship conjecture for a Kerr-Taub-NUT black hole with a test scalar field and particle. Phys. Rev. D 101(6), 064048 (2020)

49. S.J. Yang, J.J. Wan, J. Chen, J. Yang, Y.Q. Wang, Weak cosmic censorship conjecture for the novel $4 D$ charged Einstein-GaussBonnet black hole with test scalar field and particle. Eur. Phys. J. C 80(10), 937 (2020)

50. W.B. Feng, S.J. Yang, Q. Tan, J. Yang, Y.X. Liu, Overcharging a Reissner-Nordström Taub-NUT regular black hole. Sci. China Phys. Mech. Astron. 64(6), 260411 (2021)

51. F.C. Eperon, B. Ganchev, J.E. Santos, Plausible scenario for a generic violation of the weak cosmic censorship conjecture in asymptotically flat four dimensions. Phys. Rev. D 101(4), 041502 (2020)

52. J.V. Rocha, V. Cardoso, Gravitational perturbation of the BTZ black hole induced by test particles and weak cosmic censorship in AdS spacetime. Phys. Rev. D 83, 104037 (2011)

53. M. Richartz, A. Saa, Overspinning a nearly extreme black hole and the Weak Cosmic Censorship conjecture. Phys. Rev. D 78, 081503 (2008)

54. S. Hod, Cosmic censorship: formation of a shielding horizon around a fragile horizon. Phys. Rev. D 87(2), 024037 (2013)

55. K. Düztaş, İ. Semiz, Cosmic censorship, black holes and integerspin test fields. Phys. Rev. D 88(6), 064043 (2013)

56. V. Husain, S. Singh, Penrose inequality in anti-de Sitter space. Phys. Rev. D 96(10), 104055 (2017)

57. T. Crisford, G.T. Horowitz, J.E. Santos, Testing the weak gravity cosmic censorship connection. Phys. Rev. D 97(6), 066005 (2018)

58. T.Y. Yu, W.Y. Wen, Cosmic censorship and weak gravity conjecture in the Einstein-Maxwell-dilaton theory. Phys. Lett. B 781, 713-718 (2018)

59. Y. Gim, B. Gwak, Charged black hole in gravity's rainbow: violation of weak cosmic censorship. Phys. Lett. B 794, 122-129 (2019)

60. R. Ghosh, C. Fairoos, S. Sarkar, Overcharging higher curvature black holes. Phys. Rev. D 100(12), 124019 (2019)

61. J.P. Ostriker, P.J. Steinhardt, The observational case for a low density universe with a nonzero cosmological constant. Nature 377, 600-602 (1995)

62. L.M. Wang, R.R. Caldwell, J.P. Ostriker, P.J. Steinhardt, Cosmic concordance and quintessence. Astrophys. J. 530, 17-35 (2000)

63. B. Ratra, P.J.E. Peebles, Cosmological consequences of a rolling homogeneous scalar field. Phys. Rev. D 37, 3406 (1988)

64. J.A. Frieman, C.T. Hill, R. Watkins, Late time cosmological phase transitions. 1. Particle physics models and cosmic evolution. Phys. Rev. D 46, 1226-1238 (1992)

65. T. Chiba, N. Sugiyama, T. Nakamura, Cosmology with x matter. Mon. Not. R. Astron. Soc. 289, L5-L9 (1997)

66. M.S. Turner, M.J. White, CDM models with a smooth component. Phys. Rev. D 56(8), 4439 (1997)

67. R. Shaisultanov, Photon neutrino interactions in magnetic field. Phys. Rev. Lett. 80, 1586-1587 (1998)

68. M. Bucher, D.N. Spergel, Is the dark matter a solid? Phys. Rev. D 60, 043505 (1999)

69. V.V. Kiselev, Quintessence and black holes. Class. Quantum Gravity 20, 1187-1198 (2003)

70. S. Tsujikawa, Quintessence: a review. Class. Quantum Gravity 30, 214003 (2013)

71. L.H. Ford, Cosmological-constant damping by unstable scalar fields. Phys. Rev. D 35, 2339 (1987)

72. Y. Fujii, Origin of the gravitational constant and particle masses in scale invariant scalar-tensor theory. Phys. Rev. D 26, 2580 (1982) 
73. S. Chen, B. Wang, R. Su, Hawking radiation in a $d$ dimensional static spherically-symmetric black Hole surrounded by quintessence. Phys. Rev. D 77, 124011 (2008)

74. S. Fernando, Schwarzschild black hole surrounded by quintessence: null geodesics. Gen. Relativ. Gravit. 44, 18571879 (2012)

75. M. Azreg-Aï nou, M.E. Rodrigues, Thermodynamical, geometrical and Poincaré methods for charged black holes in presence of quintessence. JHEP 09, 146 (2013)

76. M. Chabab, H. El Moumni, S. Iraoui, K. Masmar, S. Zhizeh, More insight into microscopic properties of RN-AdS black hole surrounded by quintessence via an alternative extended phase space. Int. J. Geom. Methods Mod. Phys. 15(10), 1850171 (2018)

77. M. Azreg-Aï nou, Charged de Sitter-like black holes: quintessencedependent enthalpy and new extreme solutions. Eur. Phys. J. C 75(1), 34 (2015)

78. H. Ghaffarnejad, E. Yaraie, M. Farsam, Quintessence Reissner Nordström anti de Sitter black holes and joule Thomson effect. Int. J. Theor. Phys. 57(6), 1671-1682 (2018)

79. W. Hong, B. Mu, J. Tao, Thermodynamics and weak cosmic censorship conjecture in the charged RN-AdS black hole surrounded by quintessence under the scalar field. Nucl. Phys. B 949, 114826 (2019)

80. J. Liang, B. Mu, J. Tao, Thermodynamics and overcharging problem in the extended phase spaces of charged AdS black holes with cloud of strings and quintessence under charged particle absorption. Chin. Phys. C 45(2), 023121 (2021)
81. J. Liang, X. Guo, D. Chen, B. Mu, Remarks on the weak cosmic censorship conjecture of RN-AdS black holes with cloud of strings and quintessence under the scalar field. Nucl. Phys. B 965, 115335 (2021)

82. H. Ghaffarnejad, E. Yaraie, M. Farsam, Thermodynamic phase transition for quintessence dyonic anti-de Sitter black holes. Eur. Phys. J. Plus 135(2), 179 (2020)

83. H. Ghaffarnejad, M. Farsam, E. Yaraie, Effects of quintessence dark energy on the action growth and butterfly velocity. Adv. High Energy Phys. 2020, 9529356 (2020)

84. R. Yin, J. Liang, B. Mu, Stability of horizon with pressure and volume of d-dimensional charged AdS black holes with cloud of strings and quintessence. arXiv:2103.08162 [gr-qc]

85. O. Minazzoli, T. Harko, New derivation of the Lagrangian of a perfect fluid with a barotropic equation of state. Phys. Rev. D 86, 087502 (2012)

86. D. Kastor, S. Ray, J. Traschen, Enthalpy and the mechanics of AdS black holes. Class. Quantum Gravity 26, 195011 (2009)

87. V. Iyer, R.M. Wald, A comparison of Noether charge and Euclidean methods for computing the entropy of stationary black holes. Phys. Rev. D 52, 4430-4439 (1995) 